\section{Detecting microcalcifications in atherosclerotic plaques by a simple trichromic staining method for epoxy embedded carotid endarterectomies}

\author{
M. Relucenti, ${ }^{1}$ R. Heyn, ' L. Petruzziello, ${ }^{1}$ \\ G. Pugliese, ${ }^{2}$ M. Taurino, ${ }^{3}$ G. Familiari ${ }^{1}$
}

'Laboratory of Electron Microscopy

Pietro M. Motta, Department of Human

Anatomic, Histologic, Forensic and

Locomotor Apparatus Sciences Sapienza

University of Rome, Italy

'Department of Clinical Sciences, II

Medical School, S. Andrea Hospital,

Sapienza University of Rome, Italy

${ }^{3}$ Department of Vascular Surgery, II

Medical School, S. Andrea Hospital,

Sapienza University of Rome, Italy

\section{Abstract}

Atherosclerotic plaques have a high probability of undergoing rapid progression to stenosis, becoming responsible of acute coronary syndrome or stroke. Microcalcifications may act as enhancers of atherosclerotic plaque vulnerability. Considering that calcifications with a diameter smaller than $10 \mu \mathrm{m}$ in paraffin embedded tissue are rather difficult to detect, our aim was to analyze microcalcifications on semithin sections from epoxy resin embedded samples of carotid endarterectomies using an original trichromic stain (methylene blueazur B - basic fuchsine - alizarin red). We have compared samples stained either with our method, methylene blue-azur B alone or with Von Kossa staining, and methylene blue-azur B -basic fuchsine alone or with Von Kossa staining.

Our method resulted to be simple and fast (ca. $2 \mathrm{~min}$ ), it gives a sharp general contrast for all structures and allows to easy identify collagen and elastin. In addition, gray-green colour associated to intracellular lipid droplets evidences foam cells, which are particularly abundant in endarterectomies samples. Mast cells and their metachromatic granules are also well recognized. Calcifications over 0,5 $\mu \mathrm{m}$ are clearly recognizable. In conclusion, microcalcifications are clearly distinguished from the extracellular matrix in spite of their reduced dimensions. Methylene blue-azur Bbasic fuchsine-alizarin red method is easy to use, reproducible, and is particularly suitable for the identification of microcalcifications in the morphological analysis of atherosclerotic plaques.

\section{Introduction}

The term "vulnerable plaque" is used to identify thrombosis-prone plaques and plaques with a high probability of undergoing rapid progression, ${ }^{1}$ thus becoming responsible of acute coronary syndrome or stroke. The role of microcalcifications of about $10 \mu \mathrm{m}$ in diameter in atherosclerotic plaque vulnerability is still debated.$^{2-4}$ From a morphological point of view, microcalcifications are not so easy to detect, just by observing paraffin embedded histopathological (3-5 $\mu \mathrm{m}$ thick) sections. Diffuse calcification can be observed using Von Kossa staining, ${ }^{5}$ but such small calcifications cannot be accurately measured. Diffuse interstitial calcification has been analyzed by Fitzpatrick et $a l .{ }^{6}$ in coronary arteries on plastic (glycolmethylmethacrylate) embedded sections stained with Von Kossa and counterstained with Van Gieson and aldehyde fuchsine. However, these sections were $5 \mu \mathrm{m}$ thick and no measurement of the calcifications was reported. A better characterization of microcalcifications may be achieved observing at light microscopy semithin sections obtained from epoxy resin embedded tissue samples, whose thickness can be thinner than $1 \mu \mathrm{m}$. Staining of epoxy resin semithin sections can be performed by monochromic or polychromic methods on material routinely processed for transmission electron microscopy. The first staining methods were developed in the 70 's ${ }^{7,8}$ and are still very useful, both in deciding orientation of the sample prior to ultrathin sectioning and to clearly identify and describe cells, extracellular matrix, and mineral deposits. Subsequently, these polychromic stains have been applied by several authors onto epoxy embedded semithin sections. ${ }^{9-11}$

However, no polychromic method specific for our goals is available in the literature. In fact, the microscopic characterization of atherosclerotic endarterectomy specimens needs a rapid polychromic staining method allowing to clearly distinguish cells, collagen, elastin, lipids and calcium deposits. Therefore, on the basis of the method reported by D'Amico ${ }^{11}$ we have developed a simple, rapid and trichromic method, which considers the use of methylene blue- azur B, basic fuchsine and alizarin red.

\section{Materials and Methods}

\section{Sample preparation for transmis- sion electron microscopy}

Thirty endoarteriectomy specimens (20 men and 10 women, aged 63-70 years) were obtained after patients' consent. Samples
Correspondence: Dr. Michela Relucenti, Laboratory of Electron Microscopy Pietro M. Motta, Department of Human Anatomy, Sapienza University of Rome, via Alfonso Borelli 50, 00161 Rome, Italy.

Tel. +39.06 .49918012 - Fax +39.06 .49918070 .

E-mail: michela.relucenti@uniromal.it

Key words: atherosclerosis, carotid endarterectomy, calcification, polychromatic stain, epoxy resin, transmission electron microscopy.

Authors' contribution: all authors have contributed equally to this work.

Acknowledgments: the authors thank Mr. Ezio Battaglione for his technical support.

Received for publication: 18 March 2010. Accepted for publication: 3 June 2010.

This work is licensed under a Creative Commons Attribution 3.0 License (by-nc 3.0).

(C) Copyright M. Relucenti et al., 2010

Licensee PAGEPress, Italy

European Journal of Histochemistry 2010; $54: e 33$ doi:10.4081/ejh.2010.e33

belonging to atherosclerotic lesions (type III to VI) were fixed immediately upon recovery. Sample fixation time was at least 4 days at $4{ }^{\circ} \mathrm{C}$ in 2,5\% glutaraldehyde in PBS pH 7.4.

Sections were obtained from endarterectomies according to Lovett et al. ${ }^{12,13}$ Briefly, from every endarterectomy three transverse slices, about $2 \mathrm{~mm}$ thick and about 1.2-1.4 mm wide have been considered. The first slice corresponded to the maximal stenosis point, whereas the second and the third ones were 3 $\mathrm{mm}$ upstream and $3 \mathrm{~mm}$ downstream of it, respectively. The second and the third slices were cut again in three or four pieces of about $3 \mathrm{~mm}$ width. This procedure allowed obtaining parts at full thickness, with both the endothelial and tunica media sides. The slice corresponding to the maximal stenosis point was enough thick in the middle to cut multiple pieces; in this case the parts have shown the media or the endothelial side.

After at least $48 \mathrm{~h}$ samples were washed in PBS pH 7.4, cut in small pieces and postfixed in $1 \% \mathrm{OsO}_{4}$ in PBS, pH 7.4 for $2 \mathrm{~h}$. Samples were then dehydrated in a series of increasing ethanol solutions. Specimens were embedded in epoxy resin, that has been prepared with agar 100, MNA, DDSA, BDMA (Agar Scientific, Ltd., Cambridge, UK). In order to allow a correct resin penetration, samples were kept overnight in $50 \%$ propylene oxid- $50 \%$ resin. Samples were put in plastic capsules with epoxy resin and placed at $60^{\circ} \mathrm{C}$ for $48 \mathrm{~h}$ to allow resin polymerization. Extreme caution and suitable embedding capsules were used to pre- 


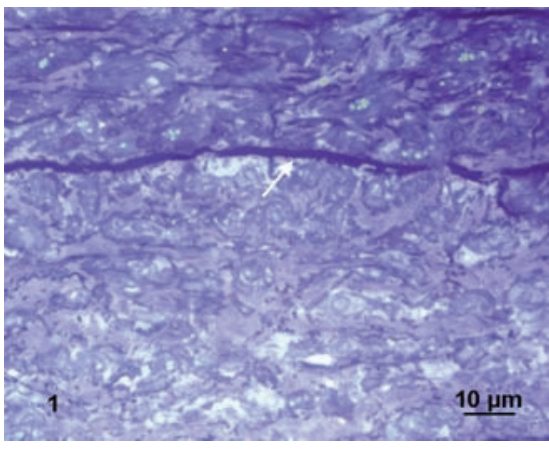

Figure 1. Carotid endarterectomy. Monochromic staining. Boundary between thickened intima and media. The extracellular matrix appears in various cyan nuances. Cells are recognizable by dark blue nucleus and light blue cytoplasm. Elastin is clearly distinguishable from collagen only when organized within the internal elastic lamina (arrow), 400X.

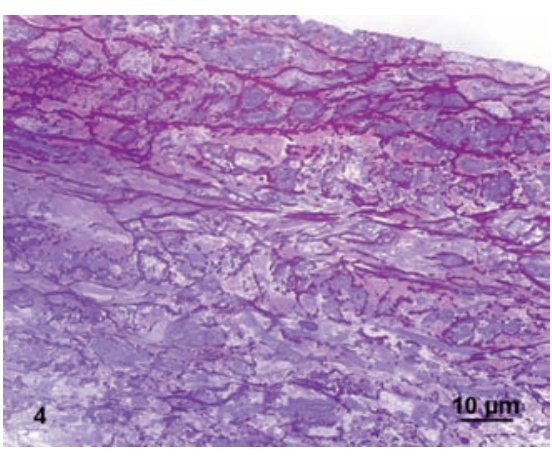

Figure 4. Carotid endarterectomy. Bichromic staining. Boundary between thickened intima and media. The use of this procedure enables a clear distinction between collagen (pink) and elastin (red violet), 400X.

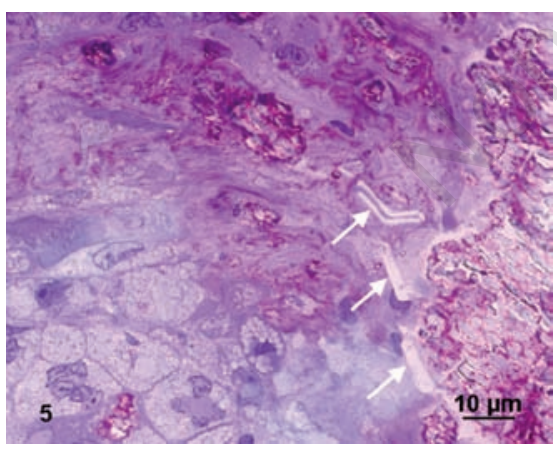

Figure 5. Carotid endarterectomy. Bichromic staining. Boundary between atherosclerotic plaque fibrous cap and necrotic core. Contrast among different types of cells and the extracellular matrix is better than in monochromic staining, 400X. Microcalcifications are not so easy to detect if very small $(<5 \mu \mathrm{m})$, because they appear very similar to collagen. Foam cells are clearly defined, with evident gray-green lipid vacuoles. Cholesterol clefts present near the large calcification are recognized (arrows), 400X.

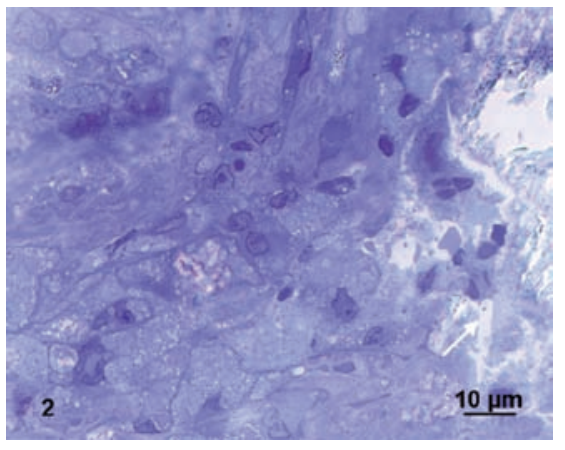

Figure 2. Carotid endarterectomy. Monochromic staining. Boundary between atherosclerotic plaque fibrous cap and necrotic core. Monochromic staining does not allow distinguishing lipid vacuoles in foam cells' cytoplasm, being confused within the general ground colour. Microcalcifications are not clearly recognized; a large calcification is shown on the right side of the micrograph. Cholesterol clefts near the large calcification are not so easy to identify (arrow), 400X.

serve the orientation of each piece during resin embedding and polymerization. Final dimensions of semithin sections, obtained with an ultramicrotome (Leica Ultracut EMUC6, Wetzlar, Germany), were about $4 \times 4$ mm (length $\mathrm{x}$ width) and $0.850 \mu \mathrm{m}$ thick, so they allow to clearly observe a full-thickness section of the slices upstream and downstream from the maximal stenosis point. Sections were placed on glass slides and dried on a hot plate at $65^{\circ} \mathrm{C}$ (LKB 2208 multiplate, Victoria, Australia). Glass slides were subsequently stained as follows.

\section{Staining solutions}

Solution A and B are prepared as reported in D'Amico. ${ }^{11}$

Solution A: methylene blue $0.5 \mathrm{~g}$, azur B $0.5 \mathrm{~g}$, borax $0.5 \mathrm{~g}$, distilled water $100 \mathrm{~mL}$;

Solution B: basic fuchsine $0.1 \mathrm{~g}, 50 \%$ ethanol $10 \mathrm{~mL}$, distilled water $10 \mathrm{~mL}$;

Solution C: $2 \%$ alizarin red in distilled water.

Staining solutions A, B, C were preserved in dark glass bottles, maintaining their stability for several months.

\section{Staining procedure}

1. Place on the glass slide just enough solution $\mathrm{A}$ in order to cover the sections, heat on a hot plate $\left(65^{\circ} \mathrm{C}\right)$ for $10 \mathrm{~s}$.

2. Rinse briefly with a jet spray of distilled water.

3. Place on the glass slide just enough solution B in order to cover the sections, heat on a hot plate $\left(65^{\circ} \mathrm{C}\right)$ for $10 \mathrm{~s}$.

4. Rinse briefly with a jet spray of distilled water.

5. Place on the glass slide just enough solu-

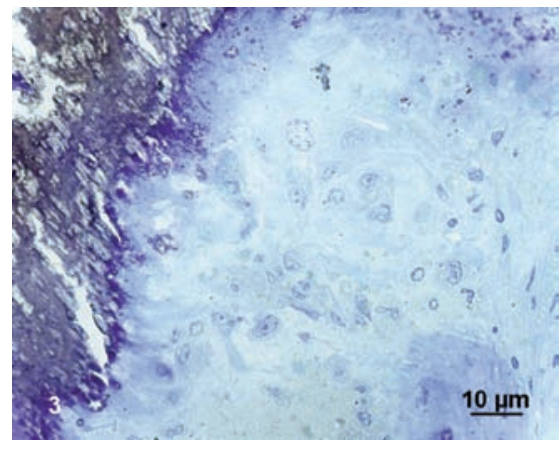

Figure 3. Carotid endarterectomy. Von Kossa staining followed by monochromic staining. The large calcification appears dark grey-black. Small calcifications are dark grey but they are very difficult to locate and to certainly identify as microcalcifications and not precipitates, because of the blue monochromic ground colour, $400 \mathrm{X}$.

tion $\mathrm{C}$ in order to cover the sections, heat on a hot plate $\left(65^{\circ} \mathrm{C}\right)$ for $10 \mathrm{~s}$.

6 . Rinse briefly with a jet spray of distilled water.

7. Dry on a hot plate.

The staining procedure was performed in three different ways:

Steps 1, 2, 7 only (monochromic stain);

Steps 1-4 and 7 only (bichromic stain);

Steps 1-7 (trichromic stain).

A section of the same sample was also stained first with Von Kossa staining and then with the monochromic stain.

A section of the same sample was also stained first with Von Kossa staining and then with the bichromic stain.

Von Kossa staining (modified from Sasaki $e t$ $\left.a l{ }^{14}\right)$ : Semithin sections were incubated with an aqueous solution of $5 \%$ silver nitrate for 15 min at room temperature under sunlight. Following distilled water rinse, the sections were incubated with a $5 \%$ sodium thiosulfate solution for $5 \mathrm{~min}$.

\section{Digital image analysis of tissue sections}

Comparisons were performed by optical quantification and counting of microcalcifications on light microscopy images captured with a camera equipped Zeiss Axioscope 40 (Oberkochen, Germany) using the MRGrab 1.0 software (Carl Zeiss Vision, Oberkochen, Germany). To achieve further information, digital images captured at low magnification were connected with the Photomerge function of Adobe Photoshop CS3 (Adobe Systems Incorporated, USA) and a complete, perfectly oriented reconstruction (with full width) was 


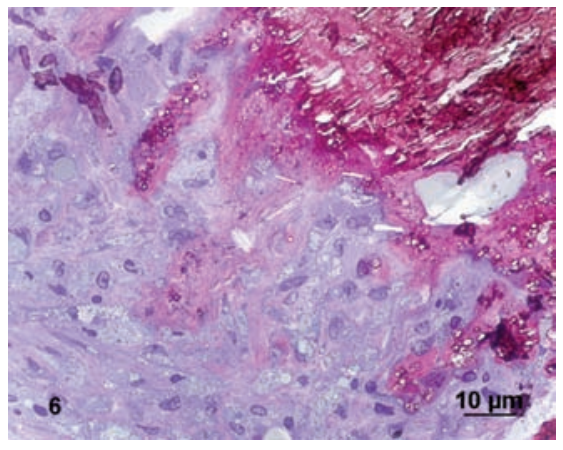

Figure 6. Carotid endarterectomy. Von Kossa staining followed by bichromic staining. Large and small calcifications appear in dark rose. It is very difficult to discriminate such calcifications because they mingle with collagen (pink), 400X.

achieved. This procedure allows recognizing the various areas of the plaque and thus making easy to further focus the subsequent observations.

\section{Statistics}

Captured images were evaluated by the same operator (M.R.) in three different occasions.

Coefficient correlation between the results obtained by analyses of Von Kossa stained and trichromic stained sections was calculated using MedCalc Software (9030 Mariakerke, Belgium); significance of the difference among groups was determined using the non-parametric MannWithney U Test (calculated using MedCalc Software, Mariakerke, Belgium) with $\mathrm{P}<0.05$ being considered statistically significant.

\section{Results}

Monochromic stain (Figures 1 and 2): this monochromic staining showed a low contrast, not allowing a clear histological characterization of cellular and extracellular components of the tissue. In fact, collagen and elastin are stained in various cyan nuances so elastin is clearly recognizable only when it is organized within the thick internal elastic lamina (Figure 1). In addition, cells that are not well highlighted in those nuclei appear dark blue (nucleolus and heterochromatin appearing darker than euchromatin), whereas the cytoplasm is shown in a lighter blue. Foam cells' cytoplasm is usually filled up of lipid droplets, so it mixed up with the general ground colour. Small calcifications are very difficult to be detected, while only larger ones are clearly recognizable (Figure 2). Cholesterol clefts did not stand out from the general ground colour.

Von Kossa stain followed by monochromic

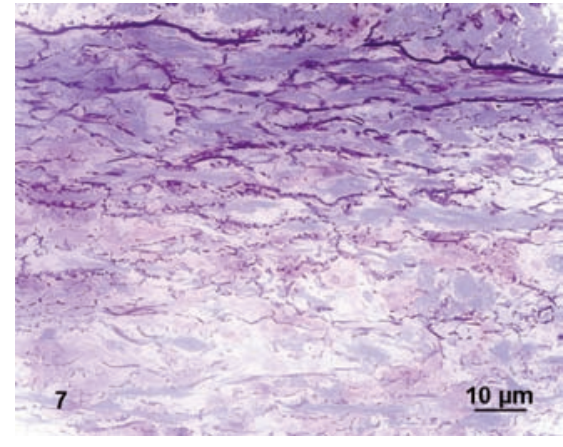

Figure 7. Carotid endarterectomy. Trichromic staining procedure. Boundary between thickened intima and media. The technique depicts a very similar image compared with Figure 4. In fact, collagen is stained in pink and elastin in purple, $400 \mathrm{X}$.

stain (Figure 3): if compared with the monochromic stain (Figures 1 and 2), the only difference appears to be the strong black stain of larger calcification. Small calcifications as well are stained dark grey-black, but they are very difficult to locate because of the blue monochromic ground colour (Figure 3).

Bichromic stain (Figures 4 and 5): the use of two dyes attempts to overcome the lack of histological contrast. This method stains collagen in pink and elastin in red violet, making the last one clearly recognizable, even when it does not organize in a thick lamina (Figure 4). Cells appear better defined, nuclear heterochromatin and nucleolus are dark blue, whereas euchromatin is lighter blue. Nuclei and cytoplasm are well contrasted; in particular, intracytoplasmic lipid droplets are graygreen (tea green). Large calcifications (over $100 \mu \mathrm{m})$, identified by their irregular shape, are fuchsia pink. Microcalcifications $(<10$ $\mu \mathrm{m})$, in turn, were not easy to detect, in particular if less than $5 \mu \mathrm{m}$, since their colour was very similar to collagen and the appearance is more homogeneous (Figure 5). Cholesterol clefts are clearly visible on the ground colour (Figure 5, arrows). The precipitation of dye was generally rare.

Von Kossa stain followed by bichromic stain (Figure 6): if compared with the bichromic stain (Figure 5), the larger calcification appears dark rose. Small calcifications are also dark rose but are very difficult to locate as they can be confused with collagen stain.

Trichromic stain (Figures 7, 8 and 9): this method uses three dyes, two as in bichromic stain with the addition of alizarin red, so collagen appears pink and elastin purple (Figure 7). Both nucleus and cytoplasm are well contrasted (Figures 8 and 9). The addition of alizarin red does not change the staining of lipid droplets, that appear gray-green (tea green) as in the bichromic stain. Calcifications, and particularly microcalcifications, are much high-

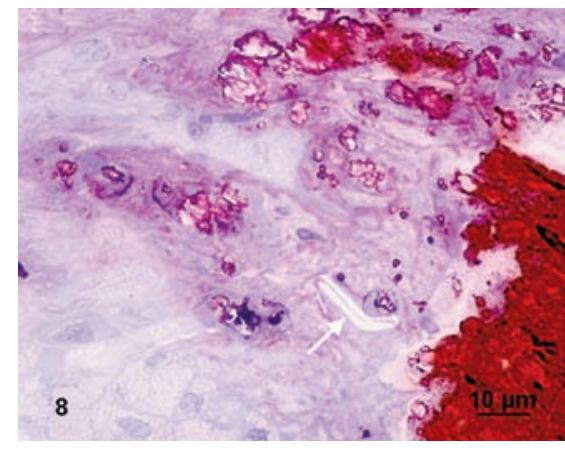

Figure 8. Carotid endarterectomy. Trichromic staining procedure. Boundary between atherosclerotic plaque fibrous cap and necrotic core. Nuclei and cytoplasm are clearly distinguished. Foam cells show sharp boundaries and defined nuclear chromatin. The addition of alizarin red does not change the staining of lipid droplets, gray-green as in the bichromic procedure. Microcalcifications are simple to detect in spite of their dimension (clearly visible and identifiable if $<2 \mu \mathrm{m}$ ), and are much highlighted by means of alizarin red. Cholesterol clefts present near the large calcification are visible (arrow), 400X. The large calcification appears more brilliant compared with Figures 3 and 6. This technique allows achieving a better contrast between microcalcifications and ground colour compared with Figures 5 and 6.

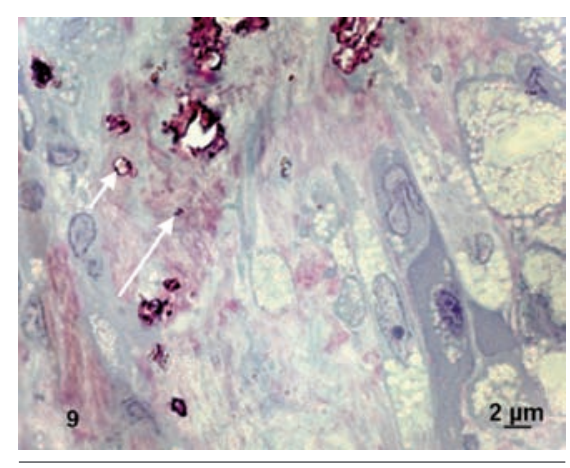

Figure 9. Carotid endarterectomy. Trichromic staining procedure. The contrast among all the elements of this trichromic image is improved. Small to very small calcifications are shown (the long arrow corresponds to the $0,5 \mu \mathrm{m}$ calcification; the short arrow corresponds to the $2 \mu \mathrm{m}$ calcification). The arrangement of fibres of the extracellular matrix is evidenced. Foam cells are clearly identified. Note the absence of precipitates, $1000 \mathrm{X}$.

lighted in red brilliant colour by means of alizarin red (Figures 8 and 9). Moreover, microcalcifications are simple to detect in spite of their dimension (Figure 9: the long arrow points out the $0,5 \mu \mathrm{m}$ calcification; the short arrow points out the $2 \mu \mathrm{m}$ calcification), keeping a different colour with respect to that of the extracellular matrix (Figures 8 and 9). This finding was further statistically con- 
firmed; in fact, the differences in the microcalcifications detection, found analyzing the three groups of images, was significant $(\mathrm{P}=0.041)$ when the operator analyzed images captured from Von Kossa stained sections, whereas it was not significant $(\mathrm{P}=0.131)$ when the images were captured from sections stained with the proposed stain.

The sharp contrast existing between structures and a very defined, resolved and polychromic image is obtained. Extracellular matrix filaments' arrangement is detectable, boundaries among cells are clean, and nuclear chromatin arrangement as well as intracytoplasmic lipid droplets can be distinguished clearly. Finally, this method enables to detect microcalcifications without precipitation of dye, as shown in Figures 7, 8 and 9.

\section{Discussion}

The thickness of the atherosclerotic plaque cap represents a critical parameter in the evaluation of vulnerable plaques. ${ }^{15}$ Recent studies suggest that the presence of microcalcifications in the cap increases plaque vulnerability, weakening the plaque and leading to its rupture, ${ }^{2,3}$ thus supporting the importance to identify this kind of calcifications. For the above reason we have developed a simple and fast procedure, which employs methylene blue, azur B, basic fuchsine and alizarin red. This staining method allows identifing and measuring microcalcifications, if over $0.5 \mu \mathrm{m}$, on epoxy resin semithin sections. The use of methylene blue, azur B and basic fuchsine allows achieving a sharp definition of the extracellular matrix, with a clear distinction between collagen and elastin. Foam cells are easily recognizable by their numerous intracytoplasmic lipid vacuoles. The advantage of our method is that it allows a clear and easy recognition of microcalcifications without the presence of precipitates. This method takes only 2 minutes, so it is fast compared with the one reported by Humphrey and Pittman, ${ }^{8}$ which lasts from $30 \mathrm{~min}$ to $1 \mathrm{~h}$ and uses two dies instead of three as in the present report. The effectiveness of our method is evident if Figures 3 and 8 are compared. Thoroughness is clear from Figure 9, in which $0.5 \mu \mathrm{m}$ and 2 $\mu \mathrm{m}$ calcifications are shown. The Von Kossa stain is considered as the gold standard in histological staining to detect calcium in cell cultures and atherosclerotic plaques. ${ }^{16}$ Nevertheless, Von Kossa staining is not specific for calcium cations and depends on the presence of the anion of the salt, which may be carbonate, phosphate, oxalate, sulphate, chloride or sulfocyanide. ${ }^{17}$ In addition, Von Kossa stain may not be sensitive enough to detect smaller and more finely distributed calcium granules. ${ }^{18}$ Moreover, the presence of phosphate do not imply the presence of hydroxyapatite or calcium. ${ }^{19}$ Atherosclerotic plaques contain bioapatite, corresponding to defect hydoxyapatite with substantial macro incorporation of carbonate, sodium, magnesium and fluoride. ${ }^{20}$ Alizarin red binds calcium, and it is very thorough, as confirmed in a confocal microscopy study on vulnerable plaque rupture. ${ }^{3}$ Although confocal microscopy allows the three dimensional reconstruction of the calcification, the image obtained is limited to the calcification itself in that cells and extracellular matrix are not visible. $^{3}$

We believe that a method with methylene blue, azur B, basic fuchsine and alizarin red, performed on epoxy resin semithin sections is particularly suitable for the detection of microcalcifications in atherosclerotic vessels, easy and reproducible. Contrary to common thinking, epoxy resin semithin sections are useful both for light and electron microscopy and for immunostaining. ${ }^{21-24}$ Besides, they can be also successfully used for in situ hybridization. ${ }^{25}$ Histological evaluation is usually based on the observation of polychromatic images taken at a magnification of about 400X. When used for the first general evaluation of a sample, transmission electron microscopy images are usually obtained at about $1250 \mathrm{X}$ and they are greyscale images. Therefore, polychromatic images obtained with the present method, at a magnification of about $1000 \mathrm{X}$, represent a bridge of information between ordinary light microscopy and transmission electron microscopy.

Although methylene blue- azur B and basic fuchsine have been used by Humphrey and Pitman, ${ }^{8}$ and alizarin red has been used by Gilmore et $a l{ }^{26}$ to stain epoxy resin embedded semithin sections, to our knowledge there is no report on such a trichromic method as the one proposed herein. In addition, our method considers the use of common laboratory dyes, easy to manage and cheap.

We propose to use this method, in studies on atherosclerotic vessels, before conventional electron microscopy. In fact, the use of convenient light microscopy techniques combined to electron microscopy can enrich and complete information achievable from clinical data and in vivo imaging techniques.

\section{References}

1. Naghavi M, Libby P, Falk E, Casscells SW, Litovsky S, Rumberger J, et al. From vulnerable plaque to vulnerable patient: a call for new definitions and risk assessment strategies: Part I. Circulation 2003;108: 1664-72.
2. Bluestein D, Alemu Y, Avrahami I, Gharib M, Dumont K, Ricotta JJ, et al. Influence of microcalcifications on vulnerable plaque mechanics using FSI modeling. J Biomech 2008;41:1111-8.

3. Vengrenyuk Y, Carlier S, Xanthos S, Cardoso L, Ganatos P, Virmani R, et al. A hypothesis for vulnerable plaque rupture due to stress-induced debonding around cellular microcalcifications in thin fibrous caps. Proc Natl Acad Sci USA 2006; 103:14678-83.

4. Vengrenyuk Y, Cardoso L, Weinbaum S. Micro-CT based analysis of a new paradigm for vulnerable plaque rupture: cellular microcalcifications in fibrous caps. Mol Cell Biomech 2008;5:37-47.

5. Jeziorska M, McCollum C, Woolley DE. Calcification in atherosclerotic plaque of human carotid arteries: associations with mast cells and macrophages. J Pathol 1998;185:10-7.

6. Fitzpatrick LA, Severson A, Edwards WD, Ingram RT. Diffuse calcification in human coronary arteries. Association of osteopontin with atherosclerosis. J Clin Invest 1994;94:1597-604.

7. Sato T, Shamoto M. A simple rapid polychrome stain for epoxy-embedded tissue. Stain Technol 1973;48:223-7.

8. Humphrey CD, Pittman FE. A simple methylene blue-azure II-basic fuchsine stain for epoxy-embedded tissue sections. Stain Technol 1974;49:9-14.

9. Litwin JA. Light microscopic histochemistry on plastic sections. Prog Histochem Cytochem 1985;16:1-84.

10. Tolivia J, Navarro A, Tolivia D. Polychromatic staining of epoxy semithin sections: a new and simple method. Histochemistry 1994;101:51-5.

11. D'Amico F. A polychromatic staining method for epoxy embedded tissue: a new combination of methylene blue and basic fuchsine for light microscopy. Biotech Histochem 2005;80:207-10.

12. Lovett JK, Redgrave JN, Rothwell PM. A critical appraisal of the performance, reporting, and interpretation of studies comparing carotid plaque imaging with histology. Stroke 2005;36:1091-7.

13. Lovett JK, Gallagher PJ, Rothwell PM. Reproducibility of histological assessment of carotid plaque: implications for studies of carotid imaging. Cerebrovasc Dis 2004;18:117-23.

14. Sasaki T, Amizuka N, Irie K, Ejiri S, Ozawa H. Localization of alkaline phosphatase and osteopontin during matrix mineralization in the developing cartilage of coccygeal vertebrae. Arch Histol Cytol 2000; 63:271-84.

15. Redgrave JN, Gallagher P, Lovett JK, 
Rothwell PM. Critical cap thickness and rupture in symptomatic carotid plaques: the Oxford plaque study. Stroke 2008;39: 1722-9.

16. Dhein S, Mohr F, Delmar M. Practical Methods in Cardiovascular Research. Springer Berlin Heidelberg, Germany, 2005, pp 485-499.

17. McGee-Russell SM. Histochemical methods for calcium. J Histochem Cytochem 1958;6:22-42.

18. Roijers RB, Dutta RK, Cleutjens JP, Mutsaers PH, de Goeij JJ, van der Vusse GJ. Early calcifications in human coronary arteries as determined with a proton microprobe. Anal Chem 2008;80:55-61.

19. Bonewald LF, Harris SE, Rosser J, Dallas MR, Dallas SL, Camacho NP, et al. Von Kossa staining alone is not sufficient to confirm that mineralization in vitro represents bone formation. Calcif Tissue Int 2003;72:537-47.

20. Tomazic BB. Physiochemical principles of cardiovascular calcification. Z Kardiol 2001;90:S68-80.

21. Cai Z, Manavis J, Cash K, Thompson PD, Blumbergs PC. Immunohistochemical staining of epoxy resin sections of peripheral nerve. Appl Immunohistochem Mol Morphol 2005;13:292-94.

22. D'Alessandro D, Mattii L, Moscato S, Bernardini N, Segnani C, Dolfi A et al. Immunohistochemical demonstration of the small GTPase RhoA on epoxy-resin embedded sections. Micron 2004;35:287-96.

23. Yano S, Kashima K, Daa T, Urabe S, Tsuji K, Nakayama I, et al. An antigen retrieval method using an alkaline solution allows immunoelectron microscopic identification of secretory granules in conventional epoxy-embedded tissue sections. J Histochem Cytochem 2003;51:199-204.

24. Groos S, Reale E, Luciano L. Re-evaluation of epoxy resin sections for light and electron microscopic immunostaining. J Histochem Cytochem 2001;49:397-406.

25. Mitrecić D, Cunko VF, Gajović S. Semi-thin sections of epoxy resin-embedded mouse embryos in morphological analysis of whole mount in situ RNA hybridization. J Microsc 2008;232:504-7.

26. Gilmore SK, Whitson SW, Bowers DE Jr. A simple method using alizarin red $\mathrm{S}$ for the detection of calcium in epoxy resin embedded tissue. Stain Technol 1986;61:89-92. 